\title{
Estimated continuous cardiac output and noninvasive stroke volume variation: pulse wave transit time (PWTT) versus arterial pulse contour analysis technique during major abdominal surgery
}

\author{
Suraphong Lorsomradee, M.D., Ph.D., Sratwadee Lorsomradee, M.D. \\ Department of Anesthesiology, Chiang Mai University Hospital, Thailand.
}

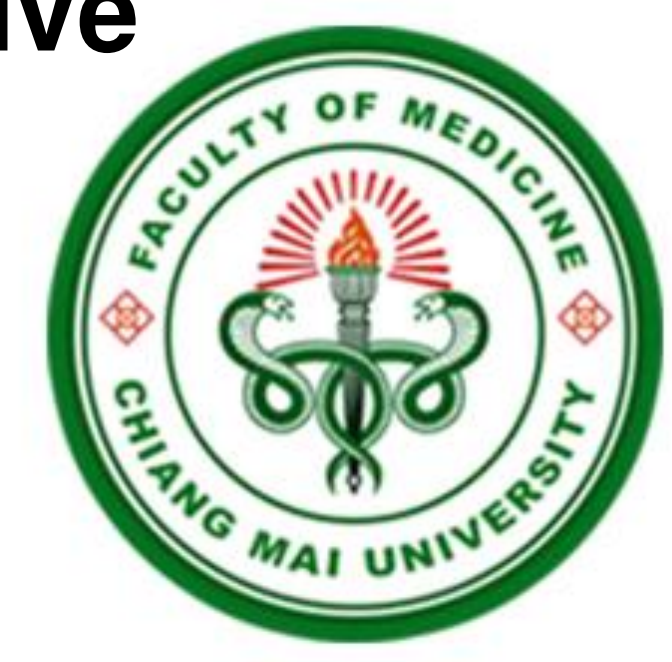

INTRODUCTION: New estimated continuous cardiac output (esCCO) uses a technique based on the relationship between pulse wave transit time (PWTT) and stroke volume. The noninvasive device provides esCCO and estimated stroke volume variation (esSVV) using routine electrocardiogram (ECG), pulse oximeter, and blood pressure. This study evaluated the overall efficacy of esCCO and esSVV using PWTT compared with the arterial pressure contour analysis (APCO) during major abdominal surgery.

MATERIALS AND METHODS: Twenty major abdominal surgery patients were received routine monitoring. The radial arterial blood pressure were attached to a sensor (FloTrac, Edwards Lifesciences, Irvine,CA) has a bifurcated cable with one going to the BSM-9101 (Nihon Kohden, Tokyo, Japan) bedside monitor to display routine arterial blood pressures, esCCO and esSVV, and

Figure1. Correlation between esCCO and APCO.

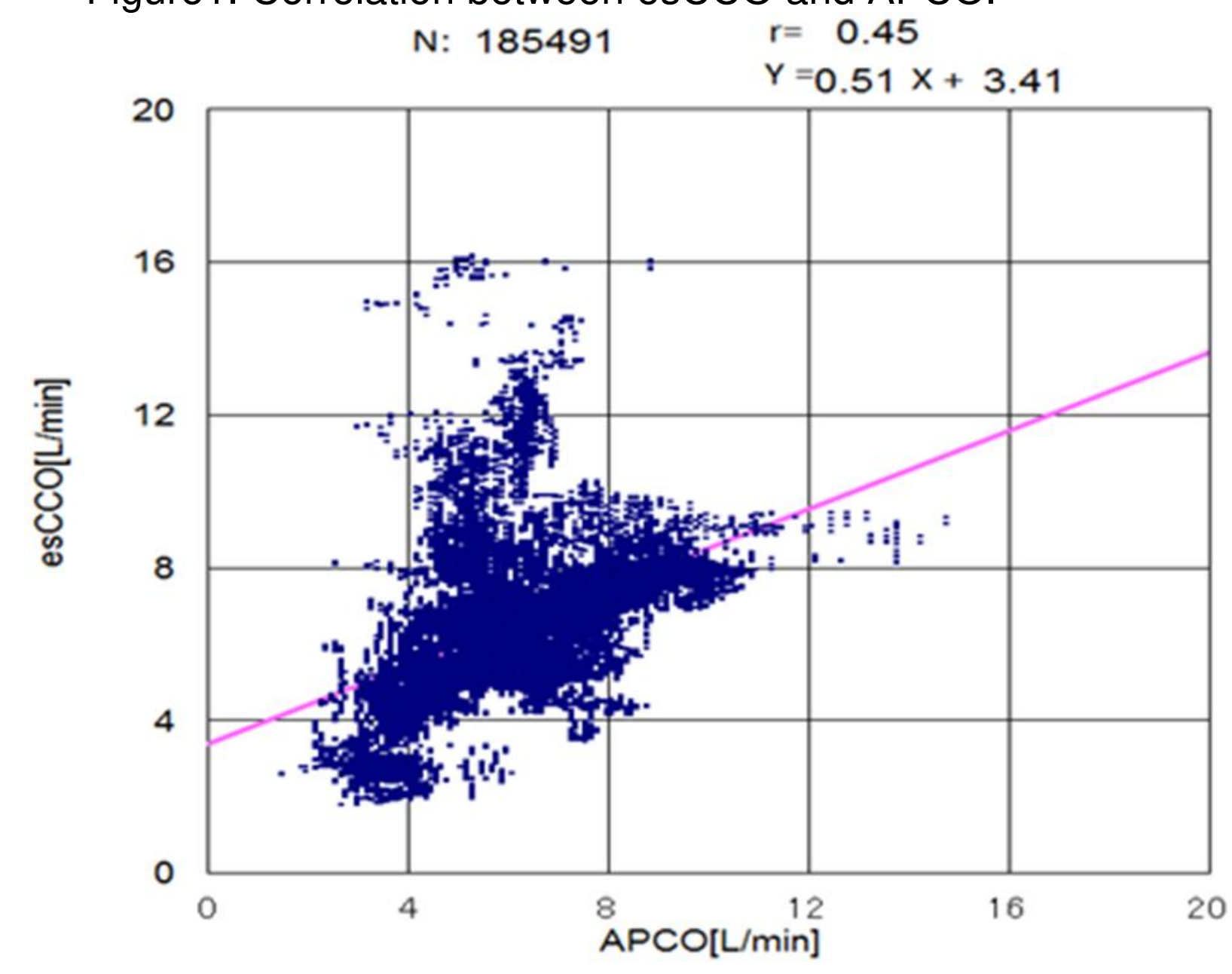

Figure2. Bland-Altman analysis showed overall agreement between esCCO and APCO. bias: 0.36 precision: 2.08

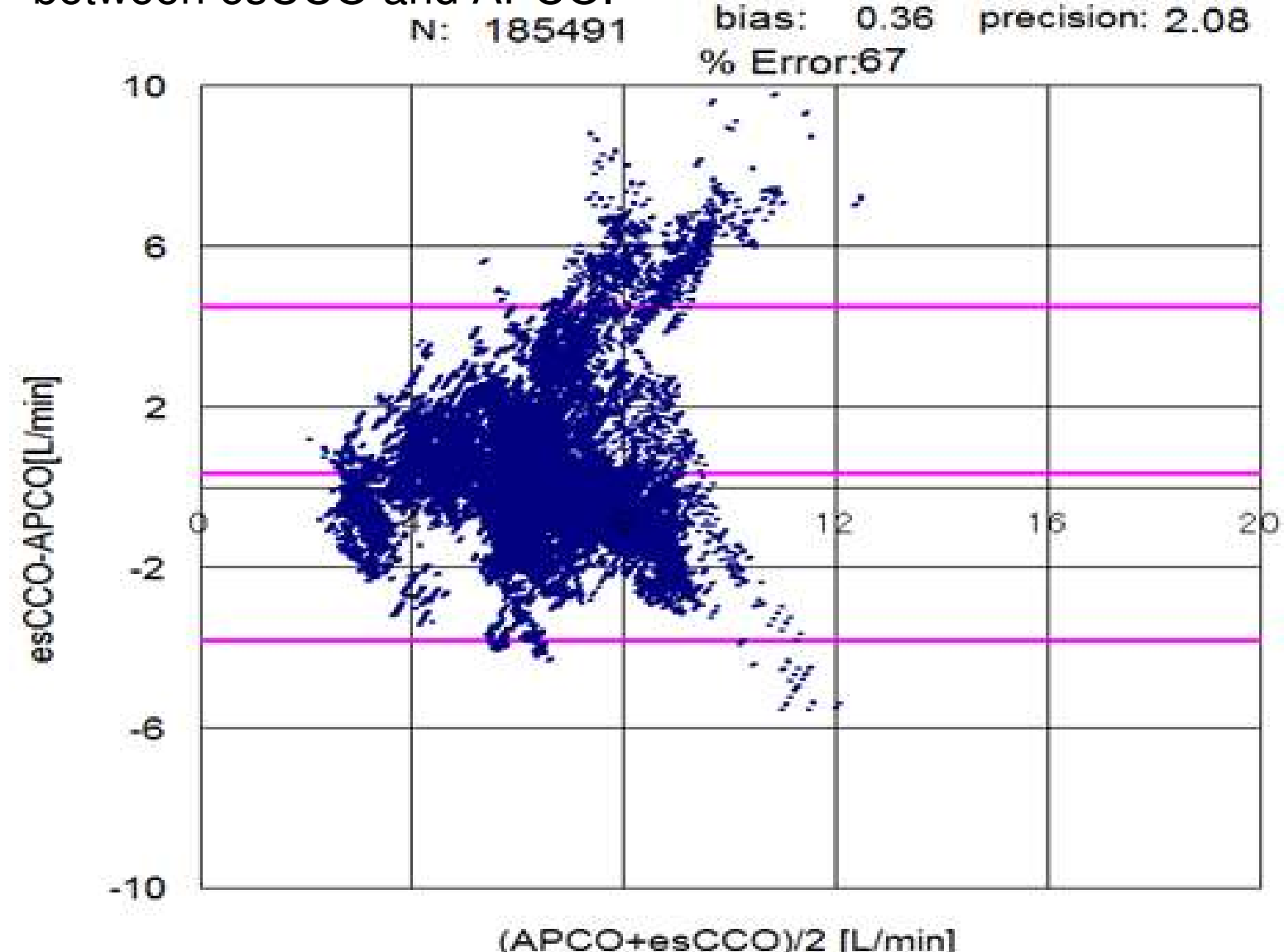

the other going to the Vigileo monitor to analyze for the APCO and SVV. All hemodynamic data including bedside monitor, vigileo APCO and esCCO will be automatically recorded simultaneously into the software during the entire operation.

RESULTS: A total of 185,491 pairs of simultaneous cardiac output measurements were recorded. The esCCO showed a poor to moderate correlation with the APCO $(R=0.45$; esCCO $=$ $\left.\left[0.51^{*} \mathrm{APCO}\right]+3.41\right)$. Bland-Altman analysis showed fairly good overall agreement between the two methods. Bias (limits of agreement) was 0.36, precision was 2.08, and \% Error was 67. However, fluid challenge test increase APCO $17.9 \% \pm 9 \%$, and increase esCCO by $10.4 \% \pm 2 \%$, whereas it decrease APSVV $40.7 \% \pm 7 \%$, and decrease esSVV by $34.7 \% \pm 27 \%$.

CONCLUSION: The esCCO showed a fairly good agreement with the APCO during perioperative major abdominal surgery. However, the trend changes of both estimated cardiac output and estimated stroke volume variation after fluid challenge test were in the same direction with the pulse contour analysis technique.

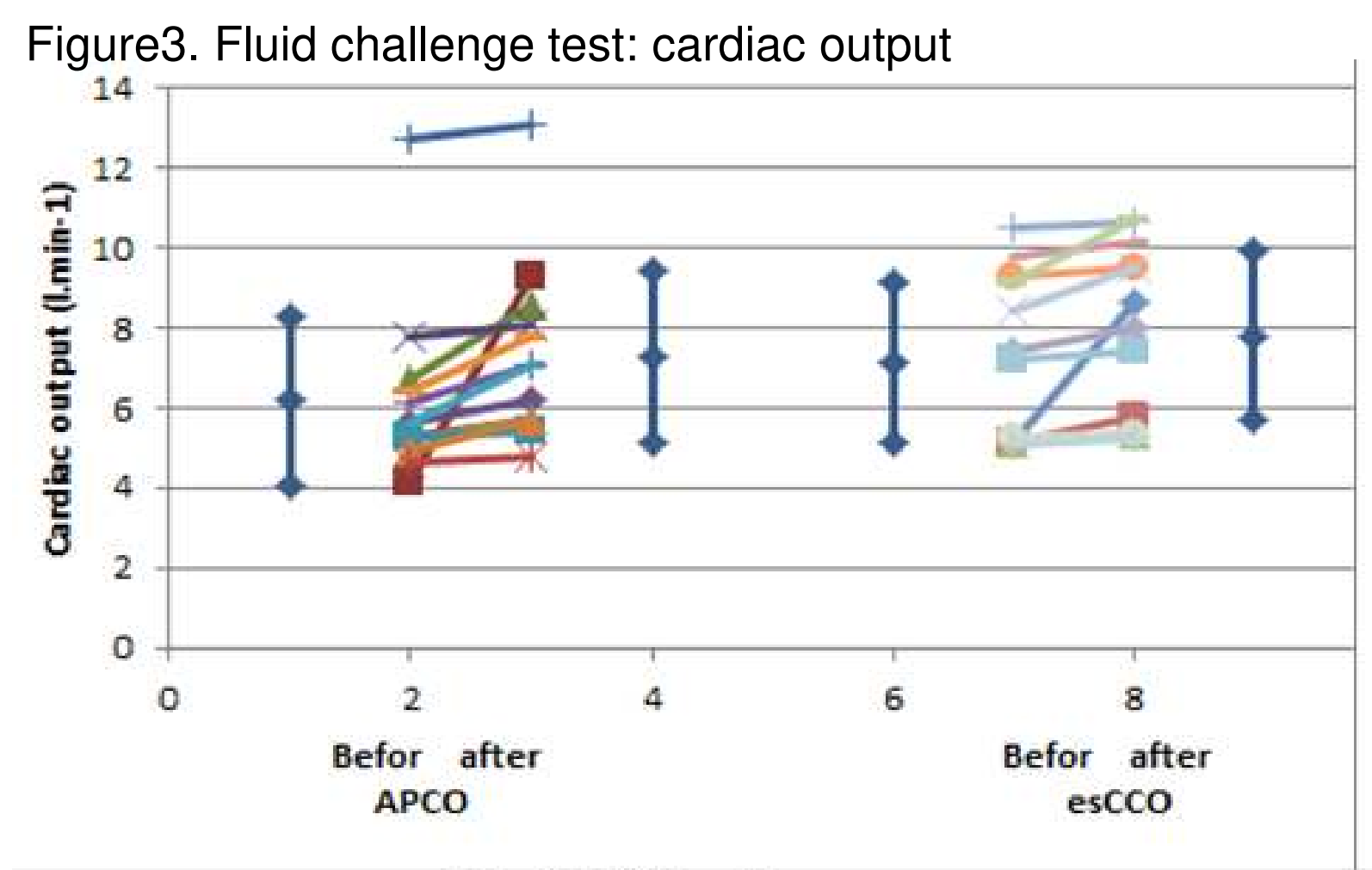

Fluid Challenge

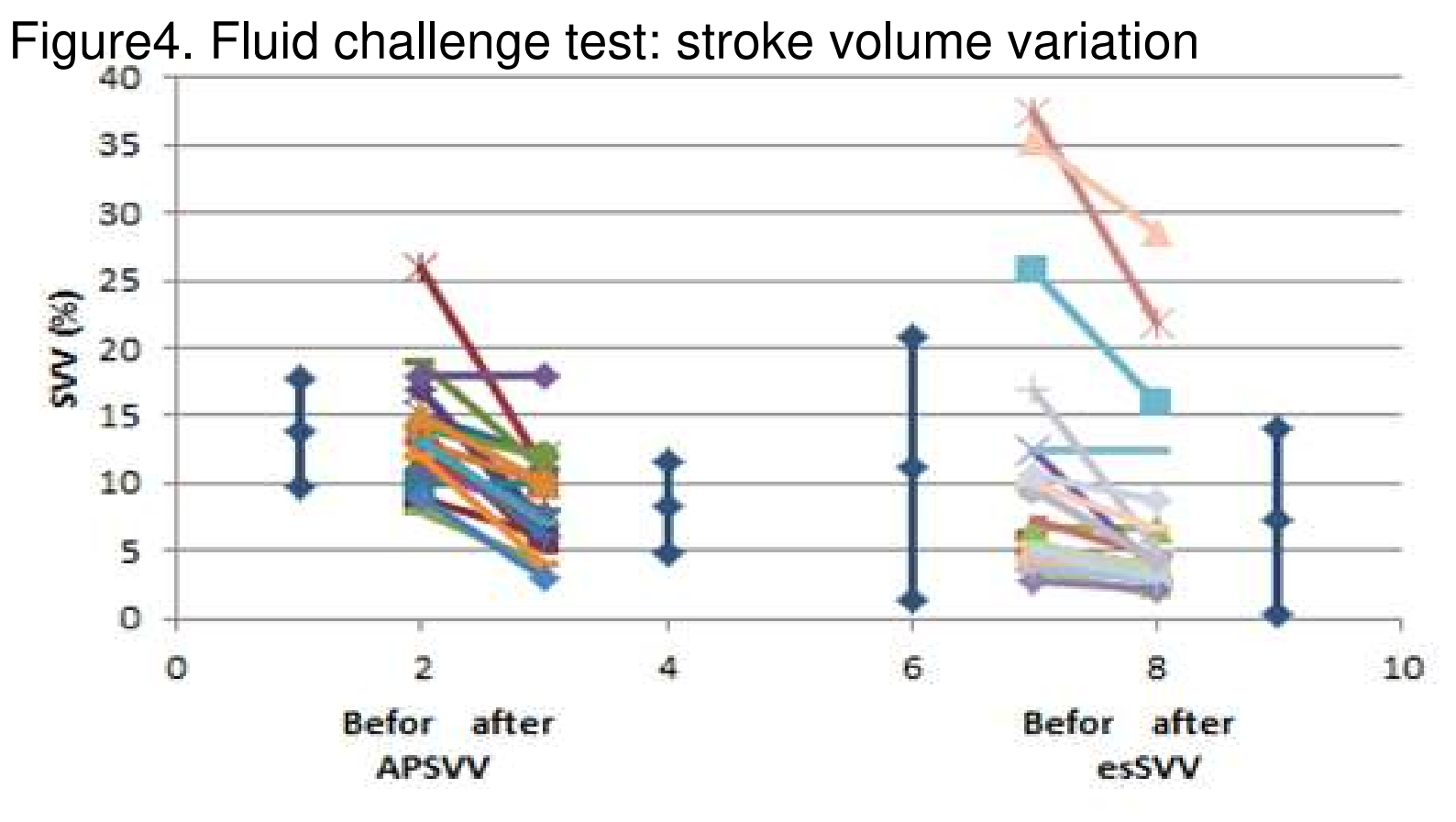

Fluid Challenge

Copyright @ 2018 Suraphong Lorsomradee MD., PhD. slorsomr@gmail.com 